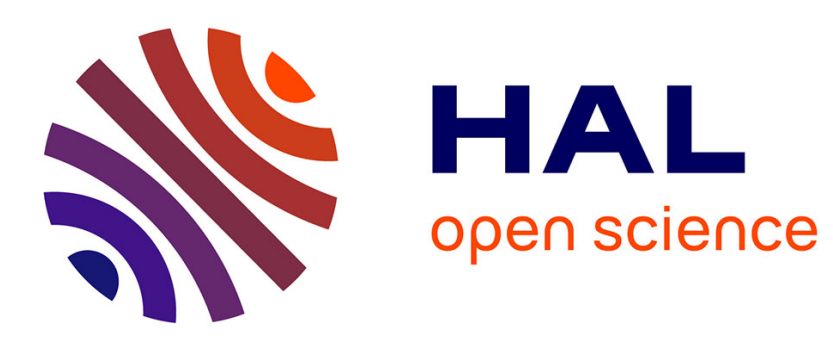

\title{
The Stationary Seasonal Hyperbolic Asymmetric Power ARCH model
}

\author{
Abdou Kâ Diongue, Dominique Guegan
}

\section{To cite this version:}

Abdou Kâ Diongue, Dominique Guegan. The Stationary Seasonal Hyperbolic Asymmetric Power ARCH model. Statistics and Probability Letters, 2007, 77 (11), pp.1158-1164. 10.1016/j.spl.2007.02.007 . halshs-00179275

\section{HAL Id: halshs-00179275 https://shs.hal.science/halshs-00179275}

Submitted on 15 Oct 2007

HAL is a multi-disciplinary open access archive for the deposit and dissemination of scientific research documents, whether they are published or not. The documents may come from teaching and research institutions in France or abroad, or from public or private research centers.
L'archive ouverte pluridisciplinaire HAL, est destinée au dépôt et à la diffusion de documents scientifiques de niveau recherche, publiés ou non, émanant des établissements d'enseignement et de recherche français ou étrangers, des laboratoires publics ou privés. 


\title{
The Stationary Seasonal Hyperbolic Asymmetric Power ARCH model
}

\author{
Abdou Kâ Diongue, Dominique Guégan ${ }^{\dagger}$
}

\begin{abstract}
More financial time series exhibit seasonality, persistence (hyperbolic decay of the autocorrelation function), asymmetric behavior and leptokurtosis. In this paper, we introduce the stationary Seasonal Hyperbolic APARCH model, which can take into account the previous features. We then investigate the probabilistic properties of the process e.g the strict and weak stationarity of the process and the long memory property.
\end{abstract}

Keywords: Seasonality, Persistence, Asymmetric, Leptokurtosis, Long memory, Hyperbolic.

\footnotetext{
${ }^{*}$ Corresponding author: Université Gaston Berger de Saint-Louis, UFR SAT, BP 234 Saint-Louis SENEGAL, e-mail: abdou.diongue@gmail.com

${ }^{\dagger}$ ENS Cachan, CES-Antenne Cachan, CES UMR 8431, Senior Academic Fellow de l'IEF, 61 avenue du président Wilson 94231 Cachan cedex, e-mail: guegan@ecogest.enscachan.fr
} 


\section{Introduction}

W andelbrot (1963) and Fama (1965) both reported evidence that large (small) changes in the prices are followed by other large (small) changes. This autocorrelation of the volatility of returns was modeled by Engle (1982) with the framework of ARCH processes (Autoregressive Conditional Heteroscedasticity) extended to GARCH models (Generalized Autoregressive Heteroscedasticity) by Bollerslev (1986).

Different studies have revealed that the $\mathrm{ARCH}$ and $\mathrm{GARCH}$ processes are unsuitable to take into account effects of asymmetry as well as the persistence noticed on the conditional volatility of stocks returns. It seems that the conditional volatility reacts more at the announcements of bad news. In particular, Black (1976) observed the existence of a negative correlation between the current return and the future volatility. Volatility asymmetry may be captured using various extensions of the GARCH model including leverage effect like the exponential GARCH (EGARCH) of Nelson (1991), the threshold ARCH (TARCH) of Zakoian (1994), the asymmetric power ARCH (APARCH) of Ding, Granger and Engle (1993) or the GJR-GARCH(1,1) introduced by Glosten, Jagannathan and Runkel (1993); the Fractionally Integrated GARCH model introduced by Baillie et al. (1996), the Asymmetric Fractionally Integrated Family GARCH (asymmetric FIFGARCH) model of Hwang (2001), the Fractionally Integrated APARCH of Tse (1998). These models allow past negative (resp. positive) shocks to have a deeper impact on current conditional volatility than past positive (resp. negative) shocks (see, among others, Black, 1976; French, Schwert and Stambaugh, 1987; Pagan and Schwert, 1990).

The increased availability of ultra-high frequency data has provided new insights for empirical analysis. One important characteristic of such data is the strong evidence of cyclical patterns in the volatility of the series, mainly due to the so-called time-of-the-day phenomena (as, for example, market open- 
ing and closing operations and lunch-hour effects). The effect of a distinct inverse-J shaped pattern in the variance of stock returns over the trading day is well studied, see, for example, Andersen and Bollerslev (1997). If this empirical evidence is neglected, Andersen and Bollerslev (1998) show that modeling and forecasting of the volatility dynamics may be seriously effected.

In this paper, we focus on a class of asymmetric long memory GARCH process that belong to the family of conditionally heteroscedastic processes . In particular, we introduce a new class of models, called the Seasonal HY-APARCH (Seasonal Hyperbolic Asymmetric Power ARCH). The problems looking stationarity of the model, existence of moments and maximum likelihood estimation method, using numerical techniques to approach the derivatives of the likelihood function with respect to parameter vector, are of the primary interest and this article explores these issues. Particularly, we examine the parameter estimation of the model when the disturbances follow a generalized hyperbolic. In section 2, we will present the S-HY-APARCH model and investigate the sufficient and necessary condition for the covariance stationary of this process. We will also look for long memory property of the model. Section 3 will study the parameter estimation of the S-HYAPARCH model when the innovations are normally distributed, t-Student or normal inverse Gaussian distributed. Section 4 concludes.

\section{S-HY-APARCH specification}

The most common definition of a long memory process is one where its autocovariance function is not absolutely summable (Baillie (1996)). The proposed S-HY-APARCH process exhibits long memory, augmenting the HYGARCH model of Davidson (2004) allowing to model asymmetric and periodic components. Assume that $\left(\varepsilon_{t}\right)_{t \in \mathbb{Z}}$ is a independently and identically distributed (i.i.d) process, $E\left(\varepsilon_{t}\right)=0$ and $\operatorname{Var}\left(\varepsilon_{t}\right)=1$. A random sequence 
$\left(x_{t}\right)_{t \in \mathbb{Z}}$ is said satisfy S-HY-APARCH model if the following equations are verified

$$
x_{t}=\nu_{t}+h_{t} \varepsilon_{t},
$$

where, for simplify, $\nu_{t}$ is equal to zero and $h_{t}$ is a time varying positive and mesureable function of the information set at time $t-1$ given by the following equation (2.3)

$$
[1-\phi(B)-\theta(B)]\left[(1-\tau)+\tau\left(1-B^{S}\right)^{d}\right] z_{t}=\omega+[1-\theta(B)] v_{t},
$$

with $\left(z_{t}\right)_{t \in \mathbb{Z}}$ a process defined by $z_{t}=\left(\left|x_{t}\right|-\eta x_{t}\right)^{\delta}, v_{t}=z_{t}-h_{t}^{\delta}, \omega>0$, $S(S \geq 0)$ represents the length of the cycle, $d(0<d<1)$ is the long memory parameter, while $\eta(|\eta|<1)$ reflects the so-called leverage effect. A positive (resp. negative) value of the $\eta$ 's means that the negative (resp. positive) shocks have a deeper impact on current conditional volatility than past positive shocks (see Black (1976)), $\delta(\delta>0)$ plays the role of a Box-Cox transformation of the conditional standard deviation and $\tau(\tau \geq 0)$ serves to eliminate the non stationarity of the process (see Davidson 2004). Let $1-\phi(B)-\theta(B)$ and $1-\theta(B)$ be the polynomials where all the roots are constrained to be outside the unit circle and defined by $\phi(B)=\sum_{i=1}^{p} \phi_{i} B^{i}$ and $\theta(B)=\sum_{j=1}^{q} \theta_{j} B^{j}$, with $p, q$ are integers. Here, B is the back shift operator defined by $B^{n} X_{t}=X_{t-n}, n \geq 0$.

Th fractional difference operator $\left(1-B^{S}\right)^{d}$ is defined by its Mclaurin series

$$
\left(1-B^{S}\right)^{d}=\sum_{j=0}^{\infty} \frac{\Gamma(j-d)}{\Gamma(-d) \Gamma(j+1)} B^{S j},
$$

where $\Gamma(x)$ is the Euler gamma function defined by $\Gamma(x)=\int_{0}^{\infty} t^{x-1} e^{-t} d t$. Rearranging the term in (2.2), an alternative representation for the S-HYAPARCH model can be obtained as,

$$
[1-\theta(B)] h_{t}^{\delta}=\omega+\left\{1-\theta(B)-\alpha(B)\left[1-\tau\left(1-\left(1-B^{S}\right)^{d}\right)\right]\right\} z_{t},
$$

where the polynomial $\alpha(B)$ is such that $\alpha(B)=1-\phi(B)-\theta(B)$.

From this representation, the conditional variance of $z_{t}$, is simply obtained 
to be

$$
h_{t}^{\delta}=\frac{\omega}{1-\theta(1)}+\left\{1-\frac{\alpha(B)}{1-\theta(B)}\left[1-\tau\left(1-\left(1-B^{S}\right)^{d}\right)\right]\right\} z_{t} .
$$

The Seasonal Hyperbolic Asymmetric Power ARCH approach enables the modeling of many features of financial market returns in the framework of stationary processes. We can note that it contains several other ARCH extensions as, among others, the linear GARCH of Bollerslev (1986), the GJR model of Glosten, Jagannathan and Runkle (1993), the TARCH of Zakoian (1994), the FIGARCH of Baillie et al. (1996), the $k$-factor GIGARCH of Guégan (2003), the FI-A-PARCH model of (Tse (1998)), the HYGARCH of Davidson (2004).

\subsection{Existence of the second order stationary solution}

One of the first questions which usually arise in the study of recursion equations of the type (2.1)-(2.3) is to find conditions for the existence of a stationary solution. We first discuss conditions on the coefficients $\psi_{j}$ and the random variables $\xi_{t}$ which guarantee the existence of the stationary solution to equations (2.1)-(2.3) with finite first and second moments.

Let $z_{t}=\left(\left|x_{t}\right|-\eta x_{t}\right)^{\delta}$ then the S-HY-APARCH process has an $\operatorname{APARCH}(0, \infty)$ representation using

$$
h_{t}^{\delta}=\psi(B) z_{t}=\psi_{0}+\sum_{j=1}^{\infty} \psi_{j} z_{t-j},
$$

with

$$
\psi(B)=\psi_{1}(B)+\psi_{2}(B),
$$

where $\psi_{1}(B)$ and $\psi_{2}(B)$ are given by the following equations

$$
\psi_{1}(B)=[I-\theta(B)]^{-1}[\omega+\phi(B)],
$$

and

$$
\psi_{2}(B)=[I-\theta(B)]^{-1}[1-\phi(B)-\theta(B)]\left[1-\left(1-B^{S}\right)^{d}\right] .
$$


For more details to the APARCH model, we can refer to Ding et al. (1993), among others.

We provide the Volterra series expansion of the process $\left(z_{t}^{\delta}\right)_{t \in \mathbb{Z}}$. Formally, from the recursion relations (2.5), we obtain

$$
h_{t}^{\delta}=\psi_{0}+\sum_{j=1}^{\infty} \psi_{j}\left(\left|\varepsilon_{t-j}\right|-\eta \varepsilon_{t-j}\right)^{\delta} h_{t-j}^{\delta} .
$$

Let $\xi_{t}$ be defined by $\xi_{t}=\left(\left|\varepsilon_{t}\right|-\eta \varepsilon_{t}\right)^{\delta}$. The recursion equation (2.5) give

$$
\begin{aligned}
h_{t}^{\delta} & =\psi_{0}+\sum_{j_{1}=1}^{\infty} \psi_{j_{1}} \xi_{t-j_{1}}\left(\psi_{0}+\sum_{j_{2}=1}^{\infty} \psi_{j_{2}} \xi_{t-j_{1}-j_{2}} h_{t-j_{1}-j_{2}}^{\delta}\right) \\
& =\psi_{0}+\sum_{j_{1}=1}^{\infty} \psi_{0} \psi_{j_{1}} \xi_{t-j_{1}}+\sum_{j_{1}=1}^{\infty} \sum_{j_{2}=1}^{\infty} \psi_{j_{1}} \psi_{j_{2}} \xi_{t-j_{1}} \xi_{t-j_{1}-j_{2}} h_{t-j_{1}-j_{2}}^{\delta} \\
& \vdots \\
& =\psi_{0} \sum_{l=0}^{\infty} \sum_{j_{1}, \cdots, j_{l}=1}^{\infty} \psi_{j_{1}} \cdots \psi_{j_{l}} \xi_{t-j_{1}} \cdots \xi_{t-j_{1}-\cdots-j_{l}} .
\end{aligned}
$$

Because $\xi_{t}=\left(\left|\varepsilon_{t}\right|-\eta \varepsilon_{t}\right)^{\delta}$, we can easily show, using equation (2.10), that the process $\left(z_{t}\right)_{t \in \mathbb{Z}}$ can be express by the following representation

$$
z_{t}=\psi_{0} \sum_{l=0}^{\infty} \sum_{j_{1}, \cdots, j_{l}=1}^{\infty} \psi_{j_{1}} \cdots \psi_{j_{l}} \xi_{t} \xi_{t-j_{1}} \cdots \xi_{t-j_{1}-\cdots-j_{l}},
$$

which can be rewritten as

$$
z_{t}=\psi_{0} \sum_{l=0}^{\infty} V_{l}(t)
$$

where $V_{0}(t)=\xi_{t}$ and for $l \geq 1$

$$
V_{l}(t)=\sum_{j_{1}, \cdots, j_{l}=1}^{\infty} \psi_{j_{1}} \cdots \psi_{j_{l}} \xi_{t} \xi_{t-j_{1}} \cdots \xi_{t-j_{1}-\cdots-j_{l}}
$$

We resume in the following Theorem 2.1 the necessary and sufficient conditions for the existence of stationary solution $(2.12),(2.1)$.

Theorem 2.1 1. If the condition

$$
E(\xi) \sum_{j=1}^{\infty} \psi_{j}<1
$$


is satisfied then the first moment of $z_{t}$ exists and is given by equation (2.14)

$$
E\left(z_{t}\right)=\frac{\psi_{0} E\left(\xi_{0}\right)}{1-E\left(\xi_{0}\right) \sum_{j=1}^{\infty} \psi_{j}}
$$

2. If the condition

$$
E\left(\xi^{2}\right)^{\frac{1}{2}} \sum_{j=1}^{\infty} \psi_{j}<1
$$

is satisfied then the second moment of $z_{t}$ exists.

Proof 2.1 By taking the unconditional expectations on both sides of (2.11) and using the independence of $\xi_{t}$ 's, we obtain

$$
\begin{aligned}
E\left(z_{t}\right) & =\psi_{0} \sum_{l=0}^{\infty} \sum_{j_{1}, \cdots, j_{l}=1}^{\infty} \psi_{j_{1}} \cdots \psi_{j_{l}} E\left(\xi_{t}\right) E\left(\xi_{t-j_{1}}\right) \cdots E\left(\xi_{t-j_{1}-\cdots-j_{l}}\right) \\
& =E\left(\xi_{t}\right) \psi_{0}\left[1+\sum_{l=1}^{\infty}\left(E\left(\xi_{0}\right) \sum_{j=1}^{\infty} \psi_{j}\right)^{l}\right] \\
& =\frac{\psi_{0} E\left(\xi_{0}\right)}{1-E\left(\xi_{0}\right) \sum_{j=1}^{\infty} \psi_{j}} .
\end{aligned}
$$

Thus, 2.14 is easily obtained.

Let us now prove the existence of the sufficient condition for the second moment of the process $\left(z_{t}\right)_{t \in \mathbb{Z}}$. applying to (2.11) the norm Minkowski inequality, we get

$$
\begin{aligned}
E\left(z_{t}^{2}\right)^{\frac{1}{2}} & \leq\left(E\left(\xi_{t}^{2}\right)^{\frac{1}{2}} \psi_{0}\right)\left\{\sum_{l=1}^{\infty} \sum_{j_{1}, \cdots, j_{l}=1}^{\infty} \psi_{j_{1}} \cdots \psi_{j_{l}}\left[E\left(\xi_{t-j_{1}}^{2}\right)\right]^{\frac{1}{2}} \cdots\left[E\left(\xi_{t-j_{1}-\cdots-j_{l}}^{2}\right)\right]^{\frac{1}{2}}\right\} \\
& =\frac{\psi_{0}\left[E\left(\xi_{0}^{2}\right)\right]^{\frac{1}{2}}}{1-\left[E\left(\xi_{0}^{2}\right)\right]^{\frac{1}{2}} \sum_{j=1}^{\infty} \psi_{j}} .
\end{aligned}
$$

Hence if condition (2.15) is satisfied, then the second moment of the process $\left(z_{t}\right)_{t \in \mathbb{Z}}$ of (2.11) is finite.

Under the conditions proved in 2.1, we investigate the strict and weak stationary solution for the S-HY-APARCH $(p, d, q, S)$ process according to $\mathrm{Gi}$ raitis et al. approach. The results are resumed in Theorem (2.2). 
Theorem 2.2 1. If equation (2.13) is verified then (2.11) is a strictly stationary solution to $z_{t}=h_{t}^{\delta} \xi_{t}$ and (2.9) with the finite first $E\left(z_{t}\right)$. Moreover, such a solution with finite first moment is a unique non anticipative solution.

2. If, in addition, equation (2.15) is verified then (2.11) is also a unique weakly stationary solution

Proof 2.2 The proof of Theorem 2.2 is similar to Theorem 2.1 in Giraitis et al. 2000.

Another way to state the stationary condition for S-HY-APARCH $(p, d, q, S)$ is to generalize Chen and An (1998) approach's for standard GARCH models. In Proposition 2.1, a necessary and sufficient condition for existence of stationary solution of the process $\left(h_{t}^{\delta}\right)_{t \in \mathbb{Z}}$ is given.

Proposition 2.1 If $\omega>0$ and the condition defined by (2.16) obtained

$$
E\left[\left(\left|\varepsilon_{t}\right|-\eta \varepsilon_{t}\right)^{\delta}\right] \sum_{j=1}^{\infty} \psi_{j}<1,
$$

then a stationary solution exists and is given by

$$
E\left(h_{t}^{\delta}\right)=\frac{\omega}{1-E\left[\left(\left|\varepsilon_{t}\right|-\eta \varepsilon_{t}\right)^{\delta}\right] \sum_{j=1}^{\infty} \psi_{j}},
$$

which depends on the probability density function of the process $\left(\varepsilon_{t}\right)_{t \in \mathbb{Z}}$.

Proof 2.3 Let $\Omega_{t-1}$ denote the information set at time $t-1$. From 2.5, the $S$-HY-APARCH $(p, d, q, S)$ process can be represented as

$$
h_{t}^{\delta}=\psi_{0}+\sum_{j=1}^{\infty} \psi_{j} Z_{t-j}
$$

Taking unconditional expectations yields

$$
\begin{aligned}
E\left(h_{t}^{\delta}\right) & =\psi_{0}+\sum_{j=1}^{\infty} \psi_{j} E\left(Z_{t-j}\right) \\
& =\psi_{0}+\sum_{j=1}^{\infty} \psi_{j} E\left[\left(\left|\varepsilon_{t-j}\right|-\eta \varepsilon_{t-j}\right)^{\delta}\right] E\left(h_{t-j}^{\delta}\right)
\end{aligned}
$$


Via the law of iterated expectations, we have

$$
\begin{aligned}
E\left(h_{t}^{\delta}\right) & =\psi_{0}+\sum_{j=1}^{\infty} \psi_{j} E_{\Omega_{t-j}}\left\{E\left[\left(h_{t-j}\left|\varepsilon_{t-j}\right|-\eta h_{t-j} \varepsilon_{t-j}\right)^{\delta}\right] \mid \Omega_{t-j}\right\} \\
& =\psi_{0}+\sum_{j=1}^{\infty} \psi_{j} E_{\Omega_{t-j}}\left\{h_{t-j}^{\delta} E\left[\left(\left|\varepsilon_{t}\right|-\eta \varepsilon_{t}\right)^{\delta}\right] \mid \Omega_{t-j}\right\} .
\end{aligned}
$$

Because $\left(\varepsilon_{t}\right)_{t \in \mathbb{Z}}$ is an independently and identically distributed process and since the process is covariance stationary, we get

$$
E\left(h_{t}^{\delta}\right)=\psi_{0}+\sum_{j=1}^{\infty} \psi_{j} E\left[\left(\left|\varepsilon_{t}\right|-\eta \varepsilon_{t}\right)^{\delta}\right] E\left(h_{t}^{\delta}\right) .
$$

Thus, we obtain (2.17).

Notice that Ding et al. (1993) derived a close form solution to $E\left[\left(\left|\varepsilon_{t}\right|-\eta \varepsilon_{t}\right)^{\delta}\right]$ in the normal case, given by:

$$
E\left[\left(\left|\varepsilon_{t}\right|-\eta \varepsilon_{t}\right)^{\delta}\right]=\frac{1}{\sqrt{2 \pi}}\left[(1+\eta)^{\delta}+(1-\eta)^{\delta}\right] 2^{\frac{\delta-1}{2}} \Gamma\left(\frac{\delta+1}{2}\right),
$$

while Lambert and Laurent (2001) obtained an analogous expression for the Student t distribution

$$
E\left[\left(\left|\varepsilon_{t}\right|-\eta \varepsilon_{t}\right)^{\delta}\right]=\left[(1+\eta)^{\delta}+(1-\eta)^{\delta}\right] \frac{\Gamma\left(\frac{\delta+1}{2}\right) \Gamma\left(\frac{\nu-\delta}{2}\right)}{2 \sqrt{(\nu-2) \pi} \Gamma\left(\frac{\nu}{2}\right)}(\nu-2)^{\frac{\delta+1}{2}}
$$

\subsection{Long memory property}

We investigate in this section, the long memory property of the S-HY$\operatorname{APARCH}(p, d, q, S)$ model. However, there are several way of characterizing long memory real valued process (see Guégan (2005), for more details). A widespread definition, in term of the autocorrelation function $\gamma(h)(h \in \mathbb{Z})$, is used here. We define a process as long memory if in the $h \rightarrow \infty$

$$
\gamma(h) \sim h^{-d} L(h)
$$

where $0<d<1$ and $L(x)$ is a slowly varying function (see Embrechts et al. (1997)) e.g $\frac{L(t x)}{L(x)} \rightarrow 1$ as $x \rightarrow \infty$. 
Let the long memory parameter $d$ is not too large, then the filter $\psi(B)$ in equation (2.6) can be closely approximated by

$$
\psi(B)=\left\{1-\frac{\alpha(B)}{1-\theta(B)}\left[1-\tau\left(1-\left(1-B^{S}\right)^{d}\right)\right]\right\} .
$$

Following Davidson (2004), we can easily show that

$$
1-\left(1-B^{S}\right)^{d}=\zeta(1+d)^{-1} \sum_{j=1}^{\infty} j^{-d-1} B^{S j}, d>0
$$

and $\zeta($.$) represents the Riemann zeta function. Thus, the hyperbolic descent$ behavior of the weights $\left(\psi_{j}\right)_{j \in \mathbb{Z}}$ can be derived. Therefore, according to the second condition for Proposition 3.2 in Giraitis et al. (2000), the presence of long memory in the process $\left(z_{t}\right)_{t \in \mathbb{Z}}$ is ensured.

\section{Parameter estimation method}

\subsection{Maximum Likelihood Estimation Method}

Let $\vartheta=\left(\omega, \phi_{1}, \cdots, \phi_{p}, \theta_{1}, \cdots, \theta_{q}\right)$ and $\gamma=\left(\vartheta^{\prime}, d, \tau, \delta, \eta\right)^{\prime}$ the vector of $(p+q+5)$ unknown parameters of the conditional dispersion equation. To estimate the S-HY-APARCH by maximum likelihood, one has to take an additional assumption on the innovation process by choosing a density, denoted $g\left(\varepsilon_{t} ; \lambda\right)$ where $\lambda$ is an extra parameter vector to be estimated. The problem to solve is to maximize the sample log-likelihood function $L_{n}(\gamma)$ for the $n$ observations $\varepsilon_{1}, \cdots, \varepsilon_{n}$, with respect to the vector of parameters $\gamma$, with $L_{n}(\gamma)$ given by the following equation (3.1)

$$
L_{n}(\gamma)=\frac{1}{n} \sum_{t=1}^{n} \log f\left(\varepsilon_{t} \mid \gamma, \Omega_{t-1}\right)
$$

where $f\left(\varepsilon_{t} \mid \gamma, \Omega_{t-1}\right)=h_{t}^{-1} g\left(\varepsilon_{t} ; \lambda\right)$. When replacing $f\left(\varepsilon_{t} \mid \gamma, \Omega_{t-1}\right)$ by its value in (3.1), we obtained

$$
L_{n}(\gamma)=-\frac{1}{n} \sum_{t=1}^{n} \log \left(h_{t}\right)+\frac{1}{n} \sum_{t=1}^{n} \log \left[g\left(\varepsilon_{t} ; \lambda\right)\right] .
$$


The maximum likelihood estimator is obtained by maximizing equation (3.2) with respect to the set of parameters $\gamma$. The score function is given by

$$
\begin{aligned}
S_{n}(\gamma) & =\frac{\partial L_{n}(\gamma)}{\partial \gamma} \\
& =\frac{1}{n} \sum_{t=1}^{n}\left[-\frac{1}{h_{t}} \frac{\partial h_{t}}{\partial \theta}+\frac{\partial \varepsilon_{t}}{\partial \theta} \frac{g^{\prime}\left(\varepsilon_{t} ; \lambda\right)}{g\left(\varepsilon_{t} ; \lambda\right)}\right] \\
& =\frac{1}{n} \sum_{t=1}^{n}\left[-\frac{1}{h_{t}} \frac{\partial h_{t}}{\partial \theta}-\frac{\varepsilon_{t}}{h_{t}} \frac{\partial h_{t}}{\partial \theta} \frac{g^{\prime}\left(\varepsilon_{t} ; \lambda\right)}{g\left(\varepsilon_{t} ; \lambda\right)}\right] \\
& =-\frac{1}{n} \sum_{t=1}^{n} \frac{1}{h_{t}} \frac{\partial h_{t}}{\partial \theta}\left[1+\varepsilon_{t} \frac{g^{\prime}\left(\varepsilon_{t} ; \lambda\right)}{g\left(\varepsilon_{t} ; \lambda\right)}\right],
\end{aligned}
$$

where $g^{\prime}$ is the derivative of $g($.$) respect to \left(\varepsilon_{t}, \lambda\right)$. The maximum likelihood estimator $\hat{\gamma}_{M L E}$ solves the system of equations $S_{n}(\theta)=0$. Since the system is highly non-linear in $\gamma$, the solution is achieved by numerical techniques such as the Berndt, Hall, Hall and Hausman (1974) algorithm.

The estimation of the parameter for a GARCH model has been studied by several authors. Lee and Hansen (1994) and Lumsdaine (1996) consider the standard normal distribution to derive the log-likelihood function in a $\operatorname{GARCH}(1,1)$ model. However, many researchers show that the high frequency financial time series exhibit thick tails behavior. To overcome this drawback, Bollerslev (1987), among others, have used the Student's t-distribution, while Nelson (1991) suggested the Generalized error Distribution. Similarly, to capture skewness, Liu and Brorsen (1995) use an asymmetric stable density. To model both skewness and kurtosis, the Normal Inverse Gaussian (NIG) was used by a number of authors (see, among others, Barndorff-Nielsen and Prause, 2001). We consider in this section, the Generalized Hyperbolic distribution in order to deal with parameter estimation of GARCH-type models. Specially, we present three particular cases of this distribution: normal distribution, Student t distribution and normal inverse Gaussian distribution. 


\subsection{The Generalized Hyperbolic distribution}

If the random variable $\varepsilon_{t}$ follows a $\mathrm{GH}$ distribution with parameters $\mu, \varpi$, $\alpha, \beta$ and $\tilde{\delta}$ which we denote $\varepsilon_{t} \sim G H(\varpi, \tilde{\delta}, \tilde{\gamma}, \alpha, \beta, \mu)$, then its density will be given by

$$
\begin{aligned}
f(x)= & \frac{\left(\frac{\tilde{\gamma}}{\tilde{\delta}}\right)^{\varpi}}{\sqrt{2 \pi K_{\lambda}(\tilde{\delta} \tilde{\gamma})}} \exp [\beta(x-\mu)] \\
& \times \frac{K_{\varpi-\frac{1}{2}}\left[\alpha \sqrt{\tilde{\delta}^{2}+(x-\mu)^{2}}\right]}{\left[\sqrt{\tilde{\delta}^{2}}+\frac{(x-\mu)^{2}}{\alpha}\right]},
\end{aligned}
$$

where $\mu(\mu \in \mathbb{R})$ and $\alpha \in \mathbb{R}$ are location parameters, $\varpi \in \mathbb{R}$ and $\tilde{\gamma} \in \mathbb{R}_{+}$ allow for flexible tail modeling, $\beta(\beta \in \mathbb{R})$ is the asymmetry parameter, $\tilde{\delta}$ $(\tilde{\delta})$ is another scale parameter and $K_{\lambda}($.$) is the modified Bessel function of$ the third kind (see, among others, Abramowitz and Stegun, 1965).

As the name suggest, it is of a very general form, being the superclass of, among others, the Student's t-distribution, the Laplace distribution, the Hyperbolic distribution, the normal inverse Gaussian distribution and the variance-gamma distribution (see Barndorff-Nilesen, 1977). In this paper, we will interest to parameter estimation of the defined $\mathrm{S}-\mathrm{HY}-\operatorname{APARCH}(p, d, q, S)$ model when the disturbances are Gaussian, Student $t$ or normal inverse Gaussian distributed.

1. The Normal distribution case If we assume that the $\varepsilon_{t}$ is normally distributed then the log-likelihood function is given by:

$$
L_{n}(\gamma)=-\frac{1}{2}\left[n \log (2 \pi)+\sum_{t=1}^{n} \log \left(h_{t}^{2}\right)+\sum_{t=1}^{n} \frac{\varepsilon_{t}^{2}}{h_{t}^{2}}\right] .
$$

The score function under Gaussianity is given by the following equation

$$
S_{n}(\gamma)=-\sum_{t=1}^{n}\left[\frac{\varepsilon_{t}}{h_{t}^{2}} \frac{\partial \varepsilon_{t}}{\partial \gamma}+\frac{1}{2} \frac{1}{h_{t}^{4}}\left(h_{t}^{2}-\varepsilon_{t}^{2}\right) \frac{\partial h_{t}^{2}}{\partial \gamma}\right] .
$$


2. The t-Student distribution case Now, if the t-Student distribution with $\nu$ degree of freedom is assumed for the disturbances $\varepsilon_{t}$ then the loglikelihood function is defined as:

$$
\begin{aligned}
L_{n}(\gamma)= & n \log \left(\frac{\Gamma\left(\frac{\nu+1}{2}\right)}{\sqrt{\pi(\nu-2)} \Gamma\left(\frac{\nu}{2}\right)}\right) \\
& -\frac{1}{2} \sum_{t=1}^{n}\left\{\log \left(h_{t}^{2}\right)+(\nu+1)\left[\log \left(1+\frac{\varepsilon_{t}^{2}}{(\nu-2) h_{t}^{2}}\right)\right]\right\}
\end{aligned}
$$

where $\Gamma$ (.) is the gamma function. The lower limit for $\nu$ is zero. For $\nu<3$, the unconditional variance does not exist. The log-likelihood function for the conditional student $t$ distribution converges to the loglikelihood function of the conditional distribution as $\nu$ tends to infinity, so that the lower $\nu$ the fatter the tails.

The score function under Student $\mathrm{t}$ distribution is given by:

$$
S_{n}(\gamma)=-\sum_{t=1}^{\infty}\left[\frac{(\nu+1) \frac{\partial \varepsilon_{t}^{2}}{\partial \gamma}}{h_{t}^{2}(\nu-2)+\varepsilon_{t}^{2}}+\frac{1}{h_{t}^{4}} \frac{\partial h_{t}^{2}}{\partial \gamma}\left(h_{t}^{2}-\frac{\nu+1}{\nu-2} \frac{\varepsilon_{t}^{2}}{1+\frac{\varepsilon_{t}^{2}}{h_{t}^{2}(\nu-2)}}\right)\right] .
$$

3. The Normal Inverse Gaussian distribution case Th NIG family of distributions is specified by four parameters. A random variable is said to be NIG distributed with parameters $\mu, \beta, \alpha$ and $\tilde{\delta}$, denoted $\varepsilon_{t} \sim$ $N I G(\alpha, \beta, \mu, \tilde{\delta})$, where $\mu$ is the location, $\beta$ the skewness, $\alpha$ the tailheaviness and $\tilde{\delta}$ the scale. The density of a $\operatorname{NIG}(\alpha, \beta, \mu, \tilde{\delta})$-variable is given by

$$
f(x, \mu, \beta, \alpha, \tilde{\delta})=\frac{\tilde{\delta} \alpha}{\pi} \exp \left[\tilde{\delta} \sqrt{\alpha^{2}-\beta^{2}}+\beta(x-\mu)\right] \frac{K_{1}[\alpha s(x-\mu)]}{s(x-\mu)},
$$

where $x \in \mathbb{R}, \mu \in \mathbb{R}, \tilde{\delta}>0,0 \leq|\beta| \leq \alpha$ and $s(x)=\sqrt{\tilde{\delta}+x^{2}}$. In particular, $\beta=0$ corresponds to a symmetric distribution. Note that here $K_{1}$ is the modified Bessel function of the third king with index 1. Specially,

$$
K_{1}(y)=\frac{y}{4} \int_{0}^{\infty} \exp \left[-\left(t+\frac{y^{2}}{4 t}\right)\right] t^{-2} d t, y \in \mathbb{R} .
$$


The log-likelihood of the $\operatorname{NIG}(\alpha, \beta, \mu, \tilde{\delta})$ distribution for the disturbances $\varepsilon_{t}$ is defined by the following equation (3.10)

$$
\begin{aligned}
L_{n}(\gamma)= & n \log (\tilde{\delta} \alpha)-n \log (\pi)+\sum_{t=1}^{n}\left[\tilde{\delta} \sqrt{\alpha^{2}-\beta^{2}}+\beta\left(\frac{\varepsilon_{t}}{h_{t}}-\mu\right)\right] \\
& +\sum_{t=1}^{n} \log \left\{\frac{K_{1}\left[\alpha s\left(\frac{\varepsilon_{t}}{h_{t}}-\mu\right)\right]}{s\left(\frac{\varepsilon_{t}}{h_{t}}-\mu\right)}\right\} .
\end{aligned}
$$

\section{Conclusion}

\section{References}

[1] Andersen, T.G. and Bollerslev, T. 1997. Intraday Periodicity and Volatility Persistence in Financial Markets. Journal of Empirical Finance 4: 115-158.

[2] Andersen, T.G. and Bollerslev, T. 1997. Heterogeneous information arrivals and return volatility dynamics: uncovering the long run in high volatility returns. Journal of Finance LII 3: 975-1005.

[3] Andersen, T.G. and Bollerslev, T. 1998. Answering the skeptics: Yes, standard volatility models do provide accurate forecasts. International Economic Review 39: 885-905.

[4] Berndt, E.R., Hall, B.H, Hall, R.E. and Hausman, J.A. 1974. Estimation and inference in nonlinear structural models. Annals of Economic and Social Measurement 3: 653-665.

[5] Barndorff-Nielsen, O.E. and Shephard, N. (2001) Econometric analysis of realized volatility and its use in estimating stochastic volatility models. Journal of the Royal Statistical Society Series B(63): 167-241. 
[6] Black, F. 1976. Studies in stock price volatility changes. Proceedings of 1976 Meeting of Business and Economic Statistics Section, American Statistical Association 177-181.

[7] Bollerslev, T. 1986. Generalized autoregressive conditional heteroscedasticity. Journal of Econometrics 31: 307-327.

[8] Bollerslev, T. 1987. A conditional heteroscedastic time series model for speculation prices and rates of return. Review of Economics and Statistics: $542-547$.

[9] Bollerslev, T. and Ghysel, E. 1996. Periodic Autoregressive Conditional Heteroscedasticity. Journal of Business 63 Economic Statistics 14(2): 139-151.

[10] Breidt, F.J., Crato, N. and Lima, P.D. 1998. The detection and the estimation of long memory in stochastic volatility. Journal of Econometrics 83: $325-348$.

[11] Dacorogna, M.M., Muller, U.A., Nagler, R.J., Olsen, R.B. and Pictet, O.V. 1993. A geographical model for the daily and weekly seasonal volatility in the foreign exchange market. Journal of International Money and Finance 12: 413-438.

[12] Ding Z., Granger C.W.J., Engle R.F. 1993. A long memory property of stock market returns and a new model. PJournal of Empirical Finance 1: $83-106$.

[13] Ding, Z and Granger, C. 1996. Modelling Volatility Persistence of Speculative Returns: A new approach. Journal of Econometrics 73: 185-215.

[14] Embrechts, P., Klüppelberg, C. and Mikosch, T. 1997. Modelling extremal events for insurance and finance. Springer-Verlag, Berlin Heidelberg. 
[15] Engle, R.F. Autoregressive conditional heteroscedasticity with estimates of the variance of U.K. inflation. Econometrica 50: 987-1008.

[16] Fama, E.F. 1965. The behavior of stock market prices. Journal of Business 38: 35:105.

[17] Fernandez, C. and Steel, M.F.J. 1998. On Bayesian modeling of fat tails and skewness. Journal of The American Statistical Association 93: 359-371.

[18] French, K., Schwert, G. and Stambaugh, R. 1987. Expected stock returns and volatility. Journal of Financial Economics 19: 3-29.

[19] Giraitis, L., Kokoszka P., Leipus R. 2000. Stationary ARCH models: Dependence structure and central limit theorem. Econometric Theory 16: $3-22$.

[20] Glosten, L., Jagannathan, R., Runkle, D. 1993. Relationship between the expected value and the volatility of the nominal excess return on stocks. Journal of Finance 48: 1779-1801.

[21] Guégan, D. 2000. A New Model: The $k$-factor GIGARCH Process. Journal of Signal Processing 4: 265-271.

[22] Guégan, D. 2003. A Prospective Study of the $k$-factor Gegenbauer Process with Heteroscedastic Errors and an Application to Inflation Rates. Finance India 17(1): 165-197.

[23] Guégan, D. 2005. How can we define the concept of long memory? An econometric survey. Econometric Reviews 24(2): 113-149.

[24] He C, Teräsvirta T. 1999a. Higher-order dependence in the general Power ARCH process and a special case. Stockholm School of Economics, Working Paper Series in Economics and Finance No. 315. 
[25] He C, Teräsvirta T. 1999b. Statistical properties of the Asymmetric Power ARCH process. Cointegration, causality, and forecasting Festschrift in honour of Clive W.J. Granger in Engle R.F. and Halbert W., oxford university press edn: chap. 19, 462-474.

[26] Modelling skewness dynamics in series of fnancial data. Discussion Paper, Institut de Statistique Louvain-la-Neuve.

[27] Lambert, P. and Laurent, S. 2001. Modelling financial time series using GARCH-type models with a skewed student distribution for the innovations. Mimeo, Université de Liége.

[28] Lee, S.W. and Hansen, B.E. 1994. Asymptotic theory for the $\operatorname{GARCH}(1,1)$ quasi-maximum likelihood estimator. Econometric Theory 10: 29-52.

[29] Liu, S. and Brorsen, B.W. 1995. Maximum likelihood estimation of a GARCH-Stable model. Journal of Applied Econometrics 10: 273-285.

[30] Lumsdaine, R.L. 1996. Consistency and asymptotic normality of the quasi-maximum likelihood estimator in $\operatorname{IGARCH}(1,1)$ and covariance stationary GARCH $(1,1)$ models. Econometrica 64(3): 575-596.

[31] Mandelbrot, B. 1963. The variation of certain speculative prices. Journal of Business 36: 394-419.

[32] Nelson, D. 1991. Conditional heteroscedasticity in asset returns: A new approach. Econometrica 59: 347-370.

[33] Pagan, A. and Schwert, G. 1990.Alternative models for conditional stock volatility. Journal of Econometrics 45: 267-290.

[34] Schoffer O. 2003. HY-A-PARCH: A stationary A-PARCH with Long Memory. Preprint. 
[35] Schwert, W. 1990. Stock volatility and the crash of 87. Review of Financial Studies 3: 77-102.

[36] Taylor, S. 1986. Modelling financial time series. Wiley New York.

[37] Tse Y.K. 1998. The conditional heteroscedasticity of the Yen-Dollar exchange rate. Journal of Applied Econometrics 13(1): 49-55.

[38] Wood, R.A., McInish, T.H. and Ord, J.K. 1985. An investigation for transaction data for NYSE stocks. Journal of Finance 40: 723-739.

[39] Woodard, W.A., Cheng, Q.C., Gray, H. .1998. A k-factor GARMA longmemory model. Journal of Time Series Analysis 19(4): 485-504.

[40] Zakoian, J.-M. 1994. Threshold heteroscedasticity models. Journal of Economic Dynamics and Control 15: 931-955. 